\title{
BMJ Open Cohort profile: Thrombolysis in Ischemic Stroke Patients (TRISP): a multicentre research collaboration
}

\begin{abstract}
Jan F Scheitz, ${ }_{1}$ Henrik Gensicke, ${ }^{2}$ Sanne M Zinkstok, ${ }^{3}$ Sami Curtze, ${ }^{4,5}$ Marcel Arnold, ${ }^{6}$ Christian Hametner, ${ }^{7}$ Alessandro Pezzini, ${ }^{8}$ Guillaume Turc, ${ }^{9}$ Andrea Zini, ${ }^{10}$ Visnja Padjen, ${ }^{11}$ Susanne Wegener, ${ }^{12}$ Annika Nordanstig, ${ }^{13}$ Lars Kellert, ${ }^{14}$ Georg Kägi, ${ }^{15}$ Yannick Bejot, ${ }^{16}$ Patrik Michel, ${ }^{17}$ Didier Leys, ${ }^{18}$ Christian H Nolte, ${ }^{1}$ Paul J Nederkoorn, ${ }^{3}$ Stefan T Engelter, ${ }^{2,19}$ on behalf of the TRISP collaboration
\end{abstract}

To cite: Scheitz JF, Gensicke H, Zinkstok SM, et al. Cohort profile: Thrombolysis in Ischemic Stroke Patients (TRISP): a multicentre research collaboration. BMJ Open 2018;8:e023265. doi:10.1136/ bmjopen-2018-023265

- Prepublication history and additional material for this paper are published online only. To view these files, please visit the journal online (http://dx.doi org/10.1136/bmjopen-2018023265).

Received 30 March 2018 Revised 6 July 2018 Accepted 4 August 2018
Check for updates

(C) Author(s) (or their employer(s)) 2018. Re-use permitted under CC BY-NC. No commercial re-use. See rights and permissions. Published by BMJ.

For numbered affiliations see end of article.

Correspondence to Professor Stefan T Engelter; Stefan.Engelter@usb.ch

\section{ABSTRACT}

Purpose The ThRombolysis in Ischemic Stroke Patients (TRISP) collaboration aims to address clinically relevant questions about safety and outcomes of intravenous thrombolysis (IVT) and endovascular thrombectomy. The findings can provide observational information on treatment of patients derived from everyday clinical practice.

Participants TRISP is an open, investigator-driven collaborative research initiative of European stroke centres with expertise in treatment with revascularisation therapies and maintenance of hospital-based registries. Al participating centres made a commitment to prospectively collect data on consecutive patients with stroke treated with IVT using standardised definitions of variables and outcomes, to assure accuracy and completeness of the data and to adapt their local databases to answer novel research questions.

Findings to date Currently, TRISP comprises 18 centres and registers $>10000$ IVT-treated patients. Prior TRISP projects provided evidence on the safety and functional outcome in relevant subgroups of patients who were excluded, underrepresented or not specifically addressed in randomised controlled trials (ie, pre-existing disability, cervical artery dissections, stroke mimics, prior statin use), demonstrated deficits in organisation of acute stroke care (ie, IVT during non-working hours, effects of onset-to-door time on onset-to-needle time), evaluated the association between laboratory findings on outcome after IVT and served to develop risk estimation tools for prediction of haemorrhagic complications and functional outcome after IVT.

Future plans Further TRISP projects to increase knowledge of the effect and safety of revascularisation therapies in acute stroke are ongoing. TRISP welcomes participation and project proposals of further centres fulfilling the outlined requirements. In the future, TRISP will be extended to include patients undergoing endovascular thrombectomy.

\section{INTRODUCTION}

Intravenous thrombolysis (IVT) with recombinant tissue plasminogen activator (rtPA)

\section{Strengths and limitations of this study}

- The Thrombolysis in Ischemic Stroke (TRISP) collaboration offers a platform to pool individual patient data from prospective registries of patients with ischaemic stroke undergoing revascularisation therapies.

- TRISP will provide data from everyday clinical practice and address clinically important questions about safety and outcomes of patients with ischaemic stroke treated with thrombolysis who are neither covered by randomised controlled trials nor other large-scale registers.

- The large sample size (currently $>10000$ from 18 centres), high completeness of data and standardised data ascertainment are strengths of TRISP.

- Data are not monitored, treatment decisions are made individually and not randomised, and most centres do not collect data on control patients who were not treated with thrombolysis.

for ischaemic stroke markedly improves functional outcome with a strong impact of time to treatment. ${ }^{1}$ The pivotal National Institute of Neurological Disorders and Stroke (NINDS) trial in 1995 and more recently the individual patient data meta-analyses of subsequent randomised controlled trials (RCTs) proved safety and efficacy of IVT in acute ischaemic stroke. $^{23}$

Implementation of IVT in acute medical treatment for ischaemic stroke has profoundly changed clinical routine. However, selection of patients for IVT in RCTs was based on strict criteria in order to exclude patients with presumably (1) low probability of favourable outcome per se or (2) high bleeding risk. ${ }^{4}$ The current exclusion criteria listed in the American Heart Association and American Stroke Association 2013 acute stroke management guidelines are still based largely on 
the criteria listed in the NINDS trial, with only minor modifications over time. ${ }^{5}$ Many criteria addressed in the guidelines were adapted from the cardiologic literature or from basic science publications. ${ }^{5}$ This overall conservative approach in selecting patients suitable for IVT is controversial as it is often unproven whether patients not meeting the established eligibility criteria do not benefit from IVT. As a consequence, the practice pattern of IVT usage has been shown to vary largely across centres. ${ }^{6}$ Furthermore, a restrictive approach in using IVT is likely to contribute to low IVT rates, especially in centres with reduced service levels. ${ }^{7}$ Systematically ascertained, comprehensive and high-quality observational data are useful to both (1) challenge or (2) confirm the clinical usefulness of commonly used but often arbitrary eligibility criteria. Of note, approximately half of patients have at least one contraindication or warning in large dedicated stroke centres. ${ }^{8}$ This underlines that clinical reality often differs from clinical trial settings. Register-based observational data can add clinically focused information whether certain warnings from licence or variables that have not been well-studied in RCTsfor example, comedications-matter or not with regard to outcome or complications of IVT. Ideally, the results from such observational studies will be verified or falsified by RCTs. However, with few exceptions (eg, age limit) this is unlikely to happen. Thus, register-based data will reflect the highest level of evidence, available currently and in the foreseeable future. In the last few years, endovascular thrombectomy (EVT) in patients with proximal vessel occlusion has emerged as another evidence-based treatment option, and acute multimodal imaging methods challenge the classical time-window concept. ${ }^{9-11}$ This makes acute stroke care even more complex. As with IVT, the benefits of EVT in subgroups of patients under-represented in clinical trials deserve further research.

In the absence of RCT-based evidence, comprehensive observational data will also be useful for individual treatment decisions about IVT and EVT, and in evaluating processes of stroke triage and care for IVT or EVT. As a prerequisite, such data have to be based on well-maintained registries containing a large number of detailed, clearly defined and well-characterised variables. TRISP (ThRombolysis in Ischemic Stroke Patients) meets these prerequisites.

\section{COHORT DESCRIPTION}

\section{Aims and objectives of TRISP}

TRISP started as a joint initiative of 11 European stroke centres in 2010. Initiated by the stroke research team Basel, an explorative, research collaborative study was designed to study the impact of prior statin treatment on outcomes of patients with stroke receiving IVT. ${ }^{12}$ Currently, TRISP comprises 18 European stroke centres (please see online supplement for a list of contributors to the TRISP collaboration). The TRISP centres cover a distance from Scandinavia to the Mediterranean area and from Western to
Eastern Europe. TRISP centres constitute high-volume IVT centres with a record of comprehensive stroke treatment for several years. All TRISP centres offer treatment fulfilling the criteria of Stroke Centres or Stroke Units as proposed by the European Stroke Organisation (ESO). ${ }^{13}$

The major aim of TRISP is to address clinically important questions about safety and outcomes of patients with ischaemic stroke treated with IVT who are neither covered by RCTs nor other large-scale IVT registers such as the Safe Implementation of Treatment in Stroke-International Stroke Thrombolysis Register (SITS-ISTR) and Get-withthe-guidelines (GWTG) Stroke. ${ }^{145}$

\section{Design, structure and policy}

TRISP operates as an investigator-driven, open platform with a clear emphasis on clinical research and data quality. TRISP is independent from industry and a non-profit collaboration. TRISP particularly aims at supporting young researchers. Thus, with the exception of the very first paper, ${ }^{12}$ first authors of the publications invariably were young stroke physicians or $\mathrm{PhD}$ students.

There is no official chair and no official administration, board or secretary within the TRISP collaboration. Currently, TRISP is coordinated by STE (Basel) and PJN (Amsterdam) who are primary contact persons.

Prior to the start of a novel project, standardised research proposals stating hypotheses and a statistical analysis plan are circulated to all participating centres and discussed for scientific content and feasibility. If a centre agrees to participate, then the centre contributes data for the time period for which they have data on all consecutive patients for the key variables of interest (eg, usage of a certain comedication). The information of the distribution of patients stratified to centre and time period is provided as online supplemental material in the respective manuscript. If a novel variable of interest needs to be added to answer a research question, the research proposal needs to describe a definition that allows to operationalise retrospective inclusion within the existing databases.

\section{Lead of distinct project and authorship}

The researcher or researches-usually 1 or 2 (rarely 3$)-$ who had the idea and rendered the analysis proposal take(s) the lead and will get first and senior authorships. Coauthorships are attributed taking into account all aspects important for the success of a research project. This includes not only mere quantitative means (ie, number of patients contributed) but also quality of data (eg, completeness, though usually high across TRISP centres), handling and pooling of the multicentre data; maintenance of the pooled data set (including data cleaning); statistics, contribution to TRISP in general, and intellectual input in details of the design or the analyses of the research project. These criteria are suggestions and the researches taking the lead in each project take the final responsibility for the distribution of authorships. 


\section{Data collection and definitions}

Data on the characteristics of patients treated with IVT are collected prospectively by all participating centres using standardised definitions and a standardised form. Not all centres have to provide data on all variables but have given a commitment to add missing variables retrospectively, if considered relevant to answer a specific research question. The dataset includes information on patients' age, sex and treatment modality (IVT alone, IVT plus EVT, EVT alone). Date and time of stroke onset as well as onset-to-needle time and door-to-needle time are systematically collected. If the exact time of symptom onset is unknown or unwitnessed, time from last seen normal until application of IVT will be provided. Stroke aetiology will be documented in distinct categories in accordance with the classification of the Trial of Org 10172 in Acute Stroke Treatment (TOAST) and classified as due to cardioembolic sources, large-artery atherosclerosis, small-vessel disease, other determined causes, concurrent aetiology or undetermined causes. ${ }^{16}$ The identified 'other determined cause' will include dissections. Other causes will be specified as precisely as possible as free text. Patients with final diagnosis other than stroke are classified as 'stroke mimics'. Dose of rtPA as well as weight and body mass index (BMI) of the patients are documented in the majority of centres. Stroke severity at baseline is measured using the National Institutes of Health Stroke Scale (NIHSS) by experienced stroke physicians. If available, NIHSS at 24 hours after treatment and at discharge from the acute care hospital will be recorded. The functional status of the patient before the index stroke will be estimated by the stroke physicians who also indicated and applied IVT using the modified Rankin Scale (mRS). ${ }^{1718}$ This prestroke mRS scores are based on information provided by patients and next-of-kin. Pre-treatment systolic and diastolic blood pressure $(\mathrm{mm} \mathrm{Hg})$ are also entered in the dataset. In case of more than one measurement, the values as close to application of IVT as possible will be noted.

Cardiovascular and stroke risk factors include atrial fibrillation (known from medical history, detected on baseline ECG or during in-hospital cardiac monitoring), diabetes mellitus (known from medical history, fasting plasma glucose $>7.0 \mathrm{mmol} / \mathrm{L}$ or $126 \mathrm{mg} / \mathrm{dL}$, HbAlc (haemoglobin A1c) $>6.5 \%$ or pre-existing treatment with antidiabetic drugs), hypercholesterolaemia (known from medical history, low-density lipoprotein $>100 \mathrm{mg} / \mathrm{dL}(2.6 \mathrm{mmol} / \mathrm{L})$ or treatment with cholesterol-lowering drugs), hypertension (known from medical history, repeated measurement of systolic blood pressure $>140 \mathrm{~mm} \mathrm{Hg}$ and/or diastolic blood pressure $>90 \mathrm{~mm}$ $\mathrm{Hg}$ ), previous stroke (known from medical history), smoking (patients who did not currently smoke were divided in non-smokers and past smokers $\geq 2$ years) and coronary artery disease (known from medical history). ${ }^{19}$ Medical history is provided by the patients, next-of-kin or family doctors.
Laboratory measures obtained on hospital admission include glucose $(\mathrm{mmol} / \mathrm{L})$, blood cell counts (platelets, haemoglobin, leucocytes), international normalised ratio and partial thromboplastin time. In case of use of novel oral anticoagulants, specific measurements of factor Xa activity, thrombin time or plasma levels of the respective drug are recorded, if available. Renal function is quantified by the estimated glomerular filtration rate (according to Chronic Kidney Disease Epidemiology Collaboration formula) ${ }^{20}$ Comedication used at the time of hospital admission prior to IVT is recorded precisely (eg, use of antithrombotic drugs and statins). Moreover, neuroimaging findings before and after treatment are systematically ascertained and comprise imaging modality (CT versus MRI) as well as specific imaging findings like hyperdense artery sign, presence and extent of early ischaemic signs according to the Alberta Stroke Program Early CT Score, ${ }^{21}$ site of vessel occlusion, presence of tandem occlusion of ipsilateral carotid artery, recanalisation status immediately after EVT and on follow-up imaging (quantified according Thrombolysis-In-Cerebral-infarction (TICI) score, ${ }^{22}$ TICI $2 \mathrm{~b} / 3$ indicate successful recanalisation), white matter disease severity, ${ }^{23}$ presence and burden of cerebral microbleeds. ${ }^{23}$

All patients are monitored for occurrence of haemorrhagic transformation. Follow-up imaging is usually scheduled at 24-36hours after treatment or earlier in case of clinical worsening. Some centres perform follow-up imaging only in case of clinical worsening. The definition of symptomatic intracerebral haemorrhage (sICH) is in accordance with the definition used in the European Cooperative Acute Stroke Study (ECASS) II (blood at any site in the brain on the CT scan together with documentation of clinical deterioration or adverse events indicating clinical worsening or causing a decrease in the NIHSS score of 4 or more points). ${ }^{24}$ The majority of centres additionally evaluate type of haemorrhagic transformation (haemorrhagic infarction, parenchymal haemorrhage), indicate whether the bleeding occurred remotely from the infarcted area and document sICH according to NINDS, ECASS-II and SITS-MOST (Safe Implementation of Thrombolysis in Stroke-Monitoring Study) definitions. ${ }^{2} 2526$

Functional outcome at 3 months is assessed using the mRS. ${ }^{18}$ The mRS is obtained by telephone calls, postal questionnaire or outpatient visits. If patients cannot be interviewed, close relatives, nurses or family doctors are asked for disability status. More recently, TRISP has been extended to include data on EVT, too.

\section{Ethical considerations and data protection}

Each study project within TRISP has to be approved by the ethics committee in the coordination and leading centres according to local regulations. It is in the responsibility of each participating centre to fulfil the local, regional and national legal and ethical requirements. Participating centres are responsible to obtain local ethics committee approval to collect and pool anonymised data with other 
centres and to keep a local patient identifier. Once a novel project has been approved, data are forwarded to the centre leading the respective project. Only strictly anonymised data prepared to match the universal data collection sheet are accepted.

\section{Patient and public involvement}

Patients and public were not involved in the study.

\section{Findings to date}

Currently, data on IVT treatments of more than 10000 patients are available. Previous publications of TRISP (1) provided insight into safety and efficacy of IVT in subgroups of patients who were excluded (eg, patients dependent on the help of others prior to stroke), under-represented or not specifically addressed (eg, dissection as cause, impaired renal function, low platelet count, BMI, prior use of statins, serotonin reuptake inhibitors, prior use of novel oral anticoagulants) in RCTs, ${ }^{12} 17$ 27-34 (2) were helpful to evaluate processes of acute stroke care such as the meaning of the 'off-hour-thrombolysis', IVT during 'working hours' or the variable 'time' in clinical practice $^{35-37}$ and (3) served to derive, validate and compare risk scores for sICH or functional 3-month outcome. ${ }^{38-40}$

The idea of TRISP is that experienced stroke centres with a record and expertise in both (1) usage of thrombolysis and (2) maintenance of hospital-based thrombolysis databases pool their data. Usually this means, 50 IVT treatments per year and an IVT rate of $>20 \%$ of all stroke admissions. Completeness of data regarding age, sex, initial NIHSS, sICH and mRS at 3 months in $>95 \%$ of consecutive IVT-treated patients is warranted by all participating centres. In addition to the characteristics of the TRISP centres stated above, an advantage of TRISP is the availability of more additional variables than in other large-scale registers and the commitment by the collaborators to (1) accuracy and completeness of the data and to (2) the willingness to adapt the local databases and add quickly new variables retrospectively and prospectively. This enables gaining explorative insights in the putative prognostic importance of additional variables with unknown impact on outcome or risk of complications such as sICH. Strengths and limitations of TRISP in general and compared with other existing IVT registers are discussed below.

\section{Strengths and limitations}

Strengths of TRISP include (1) the high completeness of data with few missing data on 3-month outcome (all centres aim to keep this below 5\%), (2) large sample sizes (several thousands) which reduces the risk of bias and allows adjustments for confounders, (3) the systematic and standardised data ascertainment which increases the homogeneity of the study population, (4) the intrinsic motivation of the study personal leads to a high rate of completeness of ascertained data sets, contributing to a high-quality registry and (5) the dynamic nature of the TRISP database due to the commitment of the centres to adapt the local database and add variables retrospectively and prospectively. In addition, (6) a large number of variables is gathered including those with unknown prognostic importance. This allows addressing novel yet unmet research questions. Moreover, (7) pooling of individual patient data increases generalisability compared with single centre studies and (8) the fact that variables and outcomes have been collected irrespective of the present research question reduces the risk of a bias.

There are other large-scale registers such as the international SITS-ISTR and the GWTG stroke registry. These registers have an unquestioned value and have served to address several research questions with high clinical impact. The TRISP collaboration does not intend to replace these registers but rather to refine the knowledge gained by them, to provide additive information and to fill gaps not covered yet. An example is the report about the usefulness of IVT in patients dependent on the help of others prior to stroke, ${ }^{17}$ which adds to the SITS-ISTR report about pre-existing disability. ${ }^{41}$ Compared with the GWTG registry that provides short-term outcomes only, TRISP provides systematic assessment of functional outcome at 3 months and systematic ascertainment of haemorrhagic complications. GWTG is mainly intended to be a database for quality control of acute stroke care. The possibility of participating centres in TRISP to develop individual research projects yields an intrinsic motivation to maintain a high data quality. Another difference of TRISP compared with SITS-ISTR (and also GWTG) are the characteristics of the sites that contribute data. In TRISP, solely experienced centres participate, while in SITS-ISTR and GWTG also low-volume centres with fewer IVT treatments per year enter their data.

Limitations are inherent to the design of TRISP. (1) Data are derived from registers that are neither monitored nor randomised. Usually, there will be no control group without IVT which disallows the assessment of effectiveness of IVT in study populations. (2) As true for all observational studies, TRISP analyses have a higher risk of bias than RCTs. Thus, we urge to a cautious interpretation of findings and observations. (3) All TRISP centres are experienced in stroke treatment. This includes in particular the use of IVT and increasingly EVT, too. This expertise implies-as a downside-a limited generalisability of TRISP findings to all stroke providers with less expertise and less advanced setting. (4) The majority of our included patients are Caucasians. Thus, we cannot compare ethnical differences, but might have the potential to set up further collaborations in the future. (5) Centres from Eastern Europe are relatively under-represented in TRISP. We encourage participation of centres from Eastern Europe in future projects to overcome this limitation and further increase generalisability of findings from TRISP. (6) Currently, there is no 'core lab' to validate haemorrhagic complications and 3-month mRS ratings. As valid for other registries like SITS and GWTG, local interpretation of outcome data may differ between sites. Since TRISP centres are high-volume centres with long-standing 
Box 1 Universal standards and requirements for contributing to TRISP*

- Prospective registry of consecutive patients with systematic checkup of missing cases.

- Comprehensive collection of baseline characteristics according to consensus definitions stated in this manuscript.

- Prospective assessment of haemorrhagic complications (symptomatic intracerebral haemorrhage according to ECASS II criteria) and functional outcome at 3 months (according to the modified Rankin Scale; either telephone interview, postal questionnaire or clinical follow-up visit).

- Approval of institutional review board to maintain the respective IVT database and to obtain 3-month follow-up data.

- TRISP centres are comprehensive stroke centres with high-volume IVT applications and usually University hospitals or affiliated to University hospitals.

- Treatment of patients with acute ischaemic stroke with IVT according to guidelines valid at the relevant time (http://www.eso-stroke. org/eso-stroke/education/guidelines.html) or documentation of deviation therefrom.

*TRISP welcomes participation and project proposals of further centres fulfilling the commitment and the outlined requirements.

Note: ECASS II, European Cooperative Acute Stroke Study II; IVT, intravenous

thrombolysis; TRISP, ThRombolysis in Ischemic Stroke Patients.

experience in maintaining IVT databases, this bias is likely to be smaller than in these other registries.

\section{Collaboration}

The TRISP collaboration welcomes participation and project proposals of further centres fulfilling the requirements stated in box 1 . The currently participating centres have agreed to fulfil the prerequisites that are summarised in box 1. Participation in other registries does not exclude from participation in the TRIPS collaboration. In order to participate or suggest a novel project, please contact the TRISP coordinating centres Basel or Amsterdam (see corresponding author or list of contributors in the online supplements). On request, the standardised data collection form and project proposal form will be forwarded. Only strictly anonymised data are used and can be included. Data sharing is restricted to non-commercial, purely academic purposes only. Furthermore, data ownership remains at the centre/physician by whom the data were originally obtained.

\section{Outlook}

Currently, several further TRISP projects are ongoing or planned. Given the potential of brain MRI to provide more prognostic information than plain CT (eg, presence of large infarct core, presence of cerebral microbleeds),${ }^{42} 43$ centres with regular MRI-based application of IVT (ie, Berlin, Bern, Lille and Paris) can offer to pool data within the subsection 'TRISP MRI', while others (eg, Basel) can focus on advanced CT imaging (TRISP-CT).

In the light of the recent positive trials of EVT in anterior circulation stroke the subsection 'TRISP endovascular' is operating since 2018. A standardised form including additional procedure-related variables was set up and agreed on during the ESO conference in 2018.

An official meeting will be scheduled annually during the respective ESO Conference. The aim is to discuss progress of ongoing projects as well as feasibility and scientific value of new project proposals. Summarising minutes are circulated to all participating centres.

\section{Summary}

The TRISP collaboration is an open platform dedicated to conduct joint research projects in patients with ischaemic stroke treated with IVT and also with EVT in the future. TRISP aims to increase knowledge regarding safety and outcomes after IVT and EVT and to evaluate processes of care. As shown in previous publications, TRISP has the potential to provide observational information on treatment of patients derived from daily clinical practice. Prospective and standardised documentation of individual patient data according to consensus definitions is a major requirement to maintain the quality of registers that contribute to TRISP. TRISP welcomes participation and project proposals of further centres fulfilling the requirements stated above.

\section{Author affiliations}

${ }^{1}$ Department of Neurology and Center for Stroke Research, Charité-

Universitätsmedizin Berlin, Berlin, Germany

${ }^{2}$ Department of Neurology and Stroke Center, University of Basel and University Hospital Basel, Basel, Switzerland

${ }^{3}$ Department of Neurology, Academic Medical Center, Amsterdam, The Netherlands

${ }^{4}$ Department of Neurology, Helsinki University Hospital, Helsinki, Finland

${ }^{5}$ Department of Neurological Sciences, University of Helsinki, Helsinki, Finland

${ }^{6}$ Department of Neurology, Inselspital, Bern University Hospital, University of Bern, Bern, Switzerland

${ }^{7}$ Department of Neurology, University Hospital Heidelberg, Heidelberg, Germany ${ }^{8}$ Department of Clinical and Experimental Sciences, Neurology Clinic, University of Brescia, Brescia, Italy

${ }^{9}$ Université Paris Descartes Sorbonne Paris Cité, Centre Hospitalier Sainte-Anne, Paris, France

${ }^{10}$ Stroke Unit, Department of Neuroscience, Nuovo Ospedale Civile S. Agostino-

Estense, University Hospital, Modena, Italy

${ }^{11}$ Neurology Clinic, Clinical Centre of Serbia, Belgrad, Serbia

${ }^{12}$ Department of Neurology, University of Zürich, Zürich, Switzerland

${ }^{13}$ Department of Neurology, Sahlgrenska University Hospital, Gothenburg, Sweden

${ }^{14}$ Department of Neurology, Klinikum der Universität München, Ludwig-Maximilians University, München, Germany

${ }^{15}$ Department of Neurology, Kantonsspital St. Gallen, St. Gallen, Switzerland

${ }^{16}$ Dijon Stroke Registry, EA4184, University Hospital and Medical School of Dijon, University of Burgundy, Dijon, France

${ }^{17}$ Department of Neurology, Centre Hospitalier Universitaire Vaudois and University of Lausanne, Lausanne, Switzerland

${ }^{18}$ Department of Neurology, University of Lille, Lille, France

${ }^{19}$ Neurorehabilitation Unit, University of Basel and University Center for Medicine of Aging and Rehabilitation, Felix Platter Hospital, Basel, Switzerland

Contributors All authors conceived the study and developed the protocol. JFS wrote the first draft. JFS, HG, SMZ, SC, MA, CH, AP, GT, AZ, VP, SW, AN, LK, GK, YB, PM, DL, CHN, PJN, STE critically revised the manuscript for important intellectual content, were involved in study concept and design, will be involved in acquisition of data and approved the final version of the manuscript. All contributors listed in the supplemental material approved submission of the manuscript.

Funding The authors have not declared a specific grant for this research from any funding agency in the public, commercial or not-for-profit sectors. 
Competing interests JFS has received speaker honoraria from W L Gore \& Associates $\mathrm{GmbH}$ and travel support from Bayer and Boehringer-Ingelheim; HG has received research support from the Swiss National Science Foundation; AZ has received funding for speaker honoraria and consulting fees from BoehringerIngelheim and Medtronic-Covidien and consulting fees from Nestec; GK received grants from Swiss Parkinson Association, Swiss Heart Association, Swiss National Science Foundation and served on advisory boards for Boehringer-Ingelheim, Bayer, Daiichi Sankyo (Schweiz) AG, Zambon, Nestle, GE Healthcare within the last two years; YB received honoraria or consulting fees from AstraZeneca France, Daiichi-Sankyo, BMS-Pfizer, Covidiem, Bayer and MSD France; PM received research grants from the Swiss National Science Foundation, the Swiss Heart Foundation; speaker fees from Boehringer-Ingelheim, Bayer, Covidien and Stryker; honoraria from scientific advisory boards from Boehringer-Ingelheim, Bayer, Pfizer, Amgen; consulting fees from Pierre-Fabre and Astra-Zeneca; and travel support from Boehringer-Ingelheim and Bayer. All this support is received by the institution (CHUV) and is used for stroke education and research; DL participated during the last 5 years to 1 advisory boards, symposia or trials sponsored by Sanofi Aventis, BMS, Astrazeneca, Boeringher-Ingelheim, Servier, Ebewe, CoLucid Pharm, Brainsgate, Photothera, Lundbeck, GSK, Bayer, Pfizer et Allergan (honorarium paid to Adrinord or research account of the hospital) and was an associated editor of the Journal of neurology, neurosurgery and psychiatry 2004-2010 (personal financial compensation); CHN has received funding for travel or speaker honoraria from Bayer, Boehringer-Ingelheim, Takeda, and BMS/Pfizer; PJN has received consulting fees from Boehringer-Ingelheim; STE has received funding for travel or speaker honoraria from Bayer and Boehringer-Ingelheim, he has served on scientific advisory boards for Bayer, Boehringer-Ingelheim, BMS/Pfizer and Covidien and on the editorial board of Stroke. He has received an educational grant from Pfizer and research support from the Science Funds (Wissenschaftsfonds) of the University Hospital Basel, the University Basel, the Swiss Heart Foundation and the Swiss National Science Foundation.

\section{Patient consent Not required.}

Provenance and peer review Not commissioned; externally peer reviewed.

Data sharing statement Data sharing will be restricted to non-commercial and academic purposes only. Data ownership remains at the center that originally obtained the data. The centers will have to give permission for re-use of the data. Data inquires or further suggestions for analyses/participation in TRISP can be made to the corresponding author (STE) or PJN.

Open access This is an open access article distributed in accordance with the Creative Commons Attribution Non Commercial (CC BY-NC 4.0) license, which permits others to distribute, remix, adapt, build upon this work non-commercially, and license their derivative works on different terms, provided the original work is properly cited, appropriate credit is given, any changes made indicated, and the use is non-commercial. See: http://creativecommons.org/licenses/by-nc/4.0/.

\section{REFERENCES}

1. Emberson J, Lees KR, Lyden P, et al. Effect of treatment delay, age, and stroke severity on the effects of intravenous thrombolysis with alteplase for acute ischaemic stroke: a meta-analysis of individual patient data from randomised trials. Lancet 2014;384:1929-35.

2. National Institute of Neurological Disorders and Stroke rt-PA Stroke Study Group. Tissue plasminogen activator for acute ischemic stroke. N Engl J Med 1995;333:1581-8.

3. Lees KR, Emberson J, Blackwell L, et al. Effects of alteplase for acute stroke on the distribution of functional outcomes: a pooled analysis of 9 trials. Stroke 2016;47:2373-9.

4. Campbell BC, Meretoja A, Donnan GA, et al. Twenty-year history of the evolution of stroke thrombolysis with intravenous alteplase to reduce long-term disability. Stroke 2015;46:2341-6.

5. Demaerschalk BM, Kleindorfer DO, Adeoye OM, et al. Scientific rationale for the inclusion and exclusion criteria for intravenous alteplase in acute ischemic stroke: a statement for healthcare professionals from the American Heart Association/American Stroke Association. Stroke 2016;47:581-641.

6. De Los Rios F, Kleindorfer DO, Guzik A, et al. Intravenous fibrinolysis eligibility: a survey of stroke clinicians' practice patterns and review of the literature. J Stroke Cerebrovasc Dis 2014;23:2130-8.

7. Gumbinger $\mathrm{C}$, Reuter $\mathrm{B}$, Hacke W, et al. Restriction of therapy mainly explains lower thrombolysis rates in reduced stroke service levels. Neurology 2016;86:1975-83.

8. Meretoja A, Putaala J, Tatlisumak T, et al. Off-label thrombolysis is not associated with poor outcome in patients with stroke. Stroke 2010;41:1450-8.
9. Goyal M, Menon BK, van Zwam WH, et al. Endovascular thrombectomy after large-vessel ischaemic stroke: a meta-analysis of individual patient data from five randomised trials. Lancet 2016;387:1723-31.

10. Nogueira RG, Jadhav AP, Haussen DC, et al. Thrombectomy 6 to 24 Hours after Stroke with a Mismatch between Deficit and Infarct. $N$ Engl J Med 2018;378:11-21.

11. Albers GW, Marks MP, Lansberg MG. Thrombectomy for Stroke with Selection by Perfusion Imaging. N Engl J Med 2018;378:708-18.

12. Engelter ST, Soinne L, Ringleb P, et al. IV thrombolysis and statins. Neurology 2011;77:888-95.

13. Ringelstein EB, Chamorro A, Kaste M, et al. European Stroke Organisation recommendations to establish a stroke unit and stroke center. Stroke 2013;44:828-40.

14. Wahlgren N, Ahmed N, Dávalos A, et al. Thrombolysis with alteplase 3-4.5 $\mathrm{h}$ after acute ischaemic stroke (SITS-ISTR): an observational study. Lancet 2008;372:1303-9.

15. Schwamm LH, Fonarow GC, Reeves MJ, et al. Get With the Guidelines-Stroke is associated with sustained improvement in care for patients hospitalized with acute stroke or transient ischemic attack. Circulation 2009;119:107-15.

16. Adams HP, Bendixen BH, Kappelle LJ, et al. Classification of subtype of acute ischemic stroke. Definitions for use in a multicenter clinical trial. TOAST. Trial of Org 10172 in Acute Stroke Treatment. Stroke 1993;24:35-41.

17. Gensicke H, Strbian D, Zinkstok SM, et al. Intravenous Thrombolysis in Patients Dependent on the Daily Help of Others Before Stroke. Stroke 2016;47:450-6.

18. van Swieten JC, Koudstaal PJ, Visser MC, et al. Interobserver agreement for the assessment of handicap in stroke patients. Stroke 1988;19:604-7.

19. Fluri F, Hatz F, Voss B, et al. Restenosis after carotid endarterectomy: significance of newly acquired risk factors. Eur $\mathrm{J}$ Neurol 2010;17:493-8.

20. Levey AS, Stevens LA, Schmid CH, et al. A new equation to estimate glomerular filtration rate. Ann Intern Med 2009;150:604-12.

21. Barber PA, Demchuk AM, Zhang J, et al. Validity and reliability of a quantitative computed tomography score in predicting outcome of hyperacute stroke before thrombolytic therapy. ASPECTS Study Group. Alberta Stroke Programme Early CT Score. Lancet 2000;355:1670-4.

22. Zaidat OO, Yoo AJ, Khatri P, et al. Recommendations on angiographic revascularization grading standards for acute ischemic stroke: a consensus statement. Stroke 2013;44:2650-63.

23. Wardlaw JM, Smith EE, Biessels GJ, et al. Neuroimaging standards for research into small vessel disease and its contribution to ageing and neurodegeneration. Lancet Neurol 2013;12:822-38.

24. Hacke W, Kaste M, Fieschi C, et al. Randomised double-blind placebo-controlled trial of thrombolytic therapy with intravenous alteplase in acute ischaemic stroke (ECASS II). Second European-Australasian Acute Stroke Study Investigators. Lancet 1998;352:1245-51.

25. Wahlgren N, Ahmed N, Dávalos A, et al. Thrombolysis with alteplase for acute ischaemic stroke in the Safe Implementation of Thrombolysis in Stroke-Monitoring Study (SITS-MOST): an observational study. Lancet 2007;369:275-82.

26. Trouillas P, von Kummer R, von KR. Classification and pathogenesis of cerebral hemorrhages after thrombolysis in ischemic stroke. Stroke 2006;37:556-61.

27. Zinkstok SM, Vergouwen MD, Engelter ST, et al. Safety and functional outcome of thrombolysis in dissection-related ischemic stroke: a meta-analysis of individual patient data. Stroke 2011;42:2515-20.

28. Gensicke H, Zinkstok SM, Roos YB, et al. IV thrombolysis and renal function. Neurology 2013;81:1780-8.

29. Gensicke H, Wicht A, Bill O, et al. Impact of body mass index on outcome in stroke patients treated with intravenous thrombolysis. Eur J Neurol 2016;23:1705-12.

30. Scheitz JF, Seiffge DJ, Tütüncü S, et al. Dose-related effects of statins on symptomatic intracerebral hemorrhage and outcome after thrombolysis for ischemic stroke. Stroke 2014;45:509-14.

31. Zinkstok SM, Engelter ST, Gensicke H, et al. Safety of thrombolysis in stroke mimics: results from a multicenter cohort study. Stroke 2013;44:1080-4.

32. Seiffge DJ, Hooff RJ, Nolte $\mathrm{CH}$, et al. Recanalization therapies in acute ischemic stroke patients: impact of prior treatment with novel oral anticoagulants on bleeding complications and outcome. Circulation 2015;132:1261-9.

33. Gensicke H, Al Sultan AS, Strbian D, et al. Intravenous thrombolysis and platelet count. Neurology 2018;90:e690-7. 
34. Scheitz JF, Turc G, Kujala L, et al. Intracerebral hemorrhage and outcome after thrombolysis in stroke patients using selective serotonin-reuptake inhibitors. Stroke 2017;48:3239-44.

35. Strbian D, Ringleb P, Michel P, et al. Ultra-early intravenous stroke thrombolysis: do all patients benefit similarly? Stroke 2013;44:2913-6.

36. Strbian D, Michel P, Ringleb P, et al. Relationship between onset-todoor time and door-to-thrombolysis time: a pooled analysis of 10 dedicated stroke centers. Stroke 2013;44:2808-13.

37. Zonneveld TP, Curtze S, Zinkstok SM, et al. Non-office-hours admission affects intravenous thrombolysis treatment times and clinical outcome. J Neurol Neurosurg Psychiatry 2017:jnnp-2017316791

38. Strbian D, Engelter S, Michel P, et al. Symptomatic intracranial hemorrhage after stroke thrombolysis: the SEDAN score. Ann Neurol 2012;71:634-41.
39. Strbian D, Meretoja A, Ahlhelm FJ, et al. Predicting outcome of IV thrombolysis-treated ischemic stroke patients: the DRAGON score. Neurology 2012;78:427-32.

40. Strbian D, Seiffge DJ, Breuer L, et al. Validation of the DRAGON score in 12 stroke centers in anterior and posterior circulation. Stroke 2013;44:2718-21.

41. Karlinski M, Kobayashi A, Czlonkowska A, et al. Role of preexisting disability in patients treated with intravenous thrombolysis for ischemic stroke. Stroke 2014;45:770-5.

42. Singer OC, Humpich MC, Fiehler J, et al. Risk for symptomatic intracerebral hemorrhage after thrombolysis assessed by diffusion-weighted magnetic resonance imaging. Ann Neurol 2008;63:52-60.

43. Dannenberg S, Scheitz JF, Rozanski M, et al. Number of cerebral microbleeds and risk of intracerebral hemorrhage after intravenous thrombolysis. Stroke 2014;45:2900-5. 